\title{
A CANONICAL EXTENSION FOR ANALYTIC FUNCTIONS ON BANACH SPACES
}

\author{
IGNACIO ZALDUENDO
}

\begin{abstract}
Given Banach spaces $E$ and $F$, a Banach space $G_{E F}$ is presented in which $E$ is embedded and which seems a natural space to which extend $F$ valued analytic functions. Any $F$-valued analytic function defined on a subset $U$ of $E$ may be extended to an open neighborhood of $U$ in $G_{E F}$. This extension generalizes that of Aron and Berner. It is also related to the Arens product in Banach algebras, to the functional calculus for bounded linear operators, and to an old problem of duality in spaces of analytic functions. A characterization of the Aron-Berner extension is given in terms of continuity properties of first-order differentials.
\end{abstract}

\section{INTRODUCTION}

The problem of extending analytic functions from a subspace $E$ of a locally convex space $G$ to the whole space $G$ was first posed by Dineen [8]. This problem is usually presented approximately in the following way: given a subspace $E$ of $G$, and an analytic function $f$ from $E$ to another locally convex space $F$, under what conditions can $f$ be extended to an analytic function $\bar{f}: G \rightarrow F$ ? There are of course various answers depending on what one considers the ingredients of the problem to be. Boland [4] has shown that if $E$ is a closed subspace of a DFN space $G$, all complex-valued analytic functions on $E$ may be extended to $G$. Aron and Berner [3] solve the problem for many $F$ 's, when there is a continuous linear extension mapping $E^{*} \rightarrow G^{*}$. In particular, Aron and Berner solve the problem in the important special case when $G=E^{* *}$ and $F$ is the complex plane $C$. Moraes [13] and others have studied the problem in various different settings.

The purpose of the present paper is to show that once one fixes Banach spaces $E$ and $F$, there is a more or less "canonical" way of choosing $G$ and of extending analytic functions to $G$. More precisely, we construct a Banach space $G_{E F}$ in which $E$ is canonically embedded and to which one may extend $F$ valued analytic functions. This extension has a universal factorization property with respect to a large class of extension maps. It is also related to an old problem of duality on spaces of analytic functions, as well as to the Arens

Received by the editors October 27, 1988.

1980 Mathematics Subject Classification (1985 Revision). Primary 46G20.

Supported by the Consejo Nacional de Investigación Cientifica y Técnica (CONICET) of the Argentine Republic. 
product in Banach algebras. The extreme cases of our construction (where $E$ or $F$ is the complex plane) give: when $F=C, G_{E F}=E^{* *}$ and the extension is that of Aron and Berner; when $E=C, G_{E F}=B(F)$ (bounded linear operators on $F$ ) and the extension is the functional calculus.

The paper is organized in the following way: in the first section we fix notation and then proceed with the definition of the extension for functions with infinite radius of uniform convergence. This is followed by a number of examples and the factorization property mentioned above. The second section is devoted to the general problem of extending arbitrary analytic functions defined on open subsets of $E$. Since our extension makes use of local Taylor expansions, we have here the problem of coherency in the "overlaps." This is related to a partial commutativity property of the Arens product. Finally, in the last section we restrict ourselves to the case $F=C$ (the Aron-Berner extension). We give a characterization of this extension in terms of continuity properties of first order differentials, and a few simple consequences.

The author would like to thank Professor T. Gamelin for introducing him to the problem, and for many helpful discussions.

\section{THE DEFINITION OF THE EXTENSION}

We first fix notation and terminology. $E$ and $F$ are complex Banach spaces. $L^{k}(E, F)\left(\right.$ resp. $\left.L_{s}^{k}(E, F)\right)$ denotes the space of all continuous (resp. continuous and symmetric) $k$-linear functions $\phi: E^{k} \rightarrow F$. These are Banach spaces with the norm

$$
\|\phi\|=\sup _{x_{1} \neq 0, \ldots, x_{k} \neq 0} \frac{\left\|\phi\left(x_{1}, \ldots, x_{k}\right)\right\|}{\left\|x_{1}\right\| \cdots\left\|x_{k}\right\|} .
$$

A continuous $k$-homogeneous polynomial is a mapping $P: E \rightarrow F$ given by $P(x)=\phi(x, \ldots, x)$, for some (unique) $\phi \in L_{s}^{k}(E, F)$. In this situation we write $P=\hat{\phi}$. We shall use the polynomial norm

$$
\|P\|_{\wp}=\sup _{x \neq 0} \frac{\|P(x)\|}{\|x\|^{k}} .
$$

For all $\phi \in L_{s}^{k}(E, F)$ one verifies $\|\hat{\phi}\|_{\wp} \leq\|\phi\| \leq\left(k^{k} / k !\right)\|\hat{\phi}\|_{\wp}$. An analytic function $f: E \rightarrow F$ is one that may locally be written as a uniformly convergent power series

$$
f(x)=\sum_{k} P_{k}(x-a) .
$$

We will denote the $k$ th order differential of $f$ at $a$ by $D^{k} f(a)$. The radius of uniform convergence at $a$ for such a power series is given by

$$
r_{a}=\frac{1}{\lim \sup _{k}\left\|P_{k}\right\|_{\wp}^{1 / k}}
$$

and is always strictly positive. Note however that it may be finite even for functions analytic on all of $E$. We shall, in this section, restrict our attention to 
functions which are analytic and bounded on balls of $E$. These have infinite radius of uniform convergence. Denote the space of all such functions $H(E, F)$. This is a Fréchet space with seminorms

$$
\|f\|_{r}=\sup _{\|x\| \leq r}\|f(x)\| .
$$

For more on analytic functions on Banach spaces see [5] or [14].

We shall also be using the Arens product in the double dual $A^{* *}$ of a commutative Banach algebra $A$ [1], [2]. This may be defined as follows: let $a, b \in A$, $\gamma \in A^{*}$, and $S, T \in A^{* *}$. One may define elements $a \gamma$ and $T_{\gamma}$ of $A^{*}$ by

$$
(a \gamma)(b)=\gamma(a b) \text { and } T_{\gamma}(a)=T(a \gamma) .
$$

Now define $S T \in A^{* *}$ by

$$
(S T)(\gamma)=S\left(T_{\gamma}\right) .
$$

This is one of the two possible Arens products in $A^{* *}$. The other is $T S$, and is, in general, different, so this product is not commutative.

Given $E$ and $F$, let

$$
G_{E F}=L(L(E, F), F) .
$$

$G_{E F}$ is a complex Banach space when endowed with the usual operator norm. When $F=C$, we have simply the double dual $E^{* *}$ of $E$. But even in the general case, one may view $E$ as a closed subspace of $G_{E F}$ via the isometric monomorphism

$$
\alpha: E \rightarrow G_{E F}
$$

given by $\alpha(x)(u)=u(x)$ for all $u \in L(E, F)$. We shall think of $E$ as contained in $G_{E F}$, and write $x$ instead of $\alpha(x)$.

We wish to extend all functions of $H(E, F)$ to the much larger space $G_{E F}$ in a more or less canonical way. The procedure will be to first extend symmetric multilinear functions; and then apply this extension to all differentials of analytic functions. We shall need the facts contained in the following trivial proposition.

Proposition 1. Let $\phi \in L_{s}^{k}(E, F), x_{1}, \ldots, x_{k} \in E$ and $T \in G_{E F}$. Then

(i) For $p=1, \ldots, k-1, \phi_{x_{1} \cdots x_{p}}: E^{k-p} \rightarrow F$ given by $\phi_{x_{1} \cdots x_{p}}\left(x_{p+1}, \ldots, x_{k}\right)$ $=\phi\left(x_{1}, \ldots, x_{k}\right)$ is a continuous symmetric $(k-p)$-linear function and $\left\|\phi_{x_{1} \cdots x_{p}}\right\|$ $\leq\|\phi\|\left\|x_{1}\right\| \cdots\left\|x_{p}\right\|$.

(ii) For $k \geq 1, \bar{T}: L_{s}^{k}(E, F) \rightarrow L_{s}^{k-1}(E, F)$ defined by $\bar{T}(\phi)\left(x_{1}, \ldots, x_{k-1}\right)$ $=T\left(\phi_{x_{1} \cdots x_{k-1}}\right)$ is a bounded linear operator and $\|\bar{T}\| \leq\|T\|$.

(iii) $T \mapsto \bar{T}$ is a bounded linear operator of norm $\leq 1$.

We may then define the following extension for symmetric $k$-linear functions. 
Definition 1. Given $\phi \in L_{s}^{k}(E, F)$, and $T_{1}, \ldots, T_{k} \in G_{E F}$,

$$
\bar{\phi}\left(T_{1}, \ldots, T_{k}\right)=\left(\bar{T}_{1} \circ \ldots \circ \bar{T}_{k}\right)(\phi) \text {. }
$$

This defines an element $\bar{\phi}$ of $L^{k}\left(G_{E F}, F\right)$. Note that $\|\bar{\phi}\| \leq\|\phi\|$. Note also that $\bar{\phi}$ is not necessarily symmetric, for in general $\bar{T}_{i}$ does not commute with $\bar{T}_{j}$. One could, in fact, define $\bar{\phi}$ in $k$ ! ways by different orderings of the $\bar{T}_{i}$ 's. We will, however, use mostly the $k$-homogeneous polynomial in $T$, $\bar{\phi}(T, \ldots, T)$, so the ordering will be immaterial. Another important case where the ordering is irrelevant is given by the following proposition.

Proposition 2. Let $T$ be any element of $G_{E F}$, and $a \in E$. Then $\bar{a} \circ \bar{T}=\bar{T} \circ \bar{a}$. Therefore in $\bar{\phi}$ one may change the order of variables belonging to $E$.

Proof. Let $\phi \in L_{s}^{k}(E, F)$, and $y_{1}, \ldots, y_{k-1} \in E$. We first prove that $\bar{a}(\phi)=$ $\phi_{a}:$

$$
\begin{aligned}
\bar{a}(\phi)\left(y_{1}, \ldots, y_{k-1}\right) & =a\left(\phi_{y_{1} \cdots y_{k-1}}\right)=\phi\left(y_{1}, \ldots, y_{k-1}, a\right) \\
& =\phi_{a}\left(y_{1}, \ldots, y_{k-1}\right)
\end{aligned}
$$

Also, $\bar{T}(\phi)_{a}=\bar{T}\left(\phi_{a}\right)$ :

$$
\begin{aligned}
\bar{T}(\phi)_{a}\left(y_{1}, \ldots, y_{k-2}\right) & =\bar{T}(\phi)\left(y_{1}, \ldots, y_{k-2}, a\right) \\
& =T\left(\phi_{y_{1} \cdots y_{k-2} a}\right)=T\left(\left(\phi_{a}\right)_{y_{1} \cdots y_{k-2}}\right) \\
& =\bar{T}\left(\phi_{a}\right)\left(y_{1}, \ldots, y_{k-2}\right)
\end{aligned}
$$

Hence, $(\bar{a} \circ \bar{T})(\phi)=\bar{a}(\bar{T}(\phi))=\bar{T}(\phi)_{a}=\bar{T}\left(\phi_{a}\right)=\bar{T}(\bar{a}(\phi))=(\bar{T} \circ \bar{a})(\phi)$.

This partial commutativity property is somewhat reminiscent to that of the Arens product, for which $a T=T a$ is valid for $a \in A$ and $T \in A^{* *}$. Another feature shared with the Arens product is a form of weak continuity of $\bar{\phi}$ in its first variable: consider, in $G_{E F}$, the weak topology induced by elements of $L(E, F)$. Then for any $u \in L(E, F), \bar{u} \in L\left(G_{E F}, F\right) \quad(\bar{u}(T)=\bar{T}(u)=T(u))$ is continuous with respect to this topology. Therefore

$$
\bar{\phi}\left(T_{1}, \ldots, T_{k}\right)=\bar{T}_{1}\left(\left(\bar{T}_{2} \circ \ldots \circ \bar{T}_{k}\right)(\phi)\right)=\overline{\left(\bar{T}_{2} \circ \cdots \circ \bar{T}_{k}\right)(\phi)}\left(T_{1}\right)
$$

is $L(E, F)$-continuous in the variable $T_{1}$.

We now define the extension.

Definition 2. Let $f \in H(E, F)$, and $T \in G_{E F}$. Then define

$$
\bar{f}(T)=\sum_{k \geq 0} \frac{1}{k !} \overline{D^{k} f(0)}(T, \ldots, T) .
$$

Proposition 3. $H(E, F) \rightarrow H\left(G_{E F}, F\right)$ given by $f \mapsto \bar{f}$ is a continuous linear extension map. 
Proof. Linearity is clear, as well as the fact that $\left.\bar{f}\right|_{E}=f$. Now take $T \in G_{E F}$. Then

$$
\begin{aligned}
\|\bar{f}(T)\| & \leq \sum_{k \geq 0}\left\|\frac{1}{k !} \overline{D^{k} f(0)}\right\|\|T\|^{k} \leq \sum_{k \geq 0}\left\|\frac{1}{k !} D^{k} f(0)\right\|\|T\|^{k} \\
& \leq \sum_{k \geq 0} \frac{k^{k}}{k !}\left\|\frac{1}{k !} D^{k} f(0)\right\|_{\wp}\|T\|^{k} \\
& \leq \sum_{k \geq 0} \frac{k^{k}\|T\|^{k}}{k ! \rho^{k}} \sup _{\|x\| \leq \rho}\|f(x)\|
\end{aligned}
$$

using the Cauchy estimates for $\left\|(1 / k !) D^{k} f(0)\right\|_{\wp}$. But $\sum k^{k}\|T\|^{k} / k ! \rho^{k}<\infty$, for $\rho>e\|T\|$. Hence, for all $r>0$,

$$
\|\bar{f}\|_{r} \leq c\|f\|_{\rho},
$$

for any $\rho>e r$.

It is clear from this proof that a function whose radius of uniform convergence at 0 is $R$ may be extended to a ball in $G_{E F}$ of radius $R^{\prime} \geq R / e$. The inequality may, in fact, be strict: for $E=C$ one trivially has $R^{\prime}=R$; when $F=C$ Davie and Gamelin have shown that this is also the case [7]. Their proof, however, is not applicable to the general case. We will return to the problem of extending functions that are not in $H(E, F)$ in $\S 2$.

Before going on with some simple properties of this extension, we want to give a few examples. In some, the definition of the extension is not easy to follow, and we do it only for some special functions.

Examples. 1. When $F=C, G_{E F}=E^{* *}$, and the extension described above is just another presentation of the Aron-Berner extension [3]. $F=C$ and $E$ reflexive is the only case in which the extension is trivial.

If $E$ is a commutative Banach algebra, and $\gamma \in E^{*}$, then $\phi: E \times E \rightarrow C$, given by $\phi(x, y)=\gamma(x y)$, is a symmetric bilinear form. Given $S, T \in E^{* *}$, the two possible extensions of $\phi$ to an element of $L^{2}\left(E^{* *}\right)$ are

$$
(\bar{S} \circ \bar{T})(\phi)=(S T)(\gamma) \text { and }(\bar{T} \circ \bar{S})(\phi)=(T S)(\gamma),
$$

where on the right-hand sides we have the Arens product defined above. Of course one may also view $\gamma(x y)$ as an analytic function on the space $E \times E$ $\left(f: E^{2} \rightarrow C / f(x, y)=\gamma(x y)\right)$. This function, when extended to $\bar{f}:\left(E^{* *}\right)^{2} \rightarrow$ $C$, produces

$$
\bar{f}(S, T)=\left(\frac{S T+T S}{2}\right)(\gamma) .
$$

This does not define a good product, however, for associativity is lost.

2. Suppose now that $E=C$. We may identify $L(C, F)$ with $F$ itself via $u \mapsto u(1) . G_{E F}$ is then $B(F)$. Take $f \in H(C, F)$. It may be written as

$$
f(z)=\sum_{k \geq 0} a_{k} z^{k}
$$


where the coefficients $a_{k}$ are elements of $F$. The extension $\bar{f}$ applied to an endomorphism $T$ of $F$ gives

$$
\bar{f}(T)=\sum_{k \geq 0} T^{k}\left(a_{k}\right) .
$$

An interesting special case occurs when the coefficients $a_{k}$ are all scalar multiples of a fixed $a \in F$ :

$$
f(z)=a \sum_{k \geq 0} \lambda_{k} z^{k}=a \cdot h(z), \quad \text { where } h: C \rightarrow C .
$$

Then

$$
\bar{f}(T)=\sum_{k \geq 0} T^{k}\left(\lambda_{k} a\right)=\left(\sum_{k \geq 0} \lambda_{k} T^{k}\right)(a)=h(T)(a) .
$$

We obtain that the functional calculus (for entire functions) is a special case of the extension defined above. This gives particular importance to the question of what the natural domain of definition is for $\bar{f}$, given an $f$ that is not entire.

3. Take $E=C^{2}$. As before, we identify $L\left(C^{2}, F\right)$ with $F^{2}$. Then $G_{E F}=$ $L\left(F^{2}, F\right)$. The function

$$
f\left(z_{1}, z_{2}\right)=a z_{1}^{2}+2 b z_{1} z_{2}+c z_{2}^{2} \quad(a, b, c \in F)
$$

extends to

$$
\bar{f}(u)=u(u(a, b), u(b, c)) .
$$

A special case: suppose $f\left(z_{1}, z_{2}\right)=\alpha z_{1} z_{2} \quad(\alpha \in F)$, and $u: F^{2} \rightarrow F$ is

$$
u(x, y)=T_{1}(x)+T_{2}(y),
$$

where $T_{i} \in B(F)$. Then

$$
\begin{aligned}
\bar{f}(u) & =u(u(0, \alpha / 2), u(\alpha / 2,0))=u\left(T_{2}(\alpha / 2), T_{1}(\alpha / 2)\right) \\
& =\frac{T_{1}\left(T_{2}(\alpha)\right)+T_{2}\left(T_{1}(\alpha)\right)}{2}=\left(\frac{T_{1} \circ T_{2}+T_{2} \circ T_{1}}{2}\right)(\alpha) .
\end{aligned}
$$

4. Surely the simplest nontrivial example is obtained by setting $E=C$, and $F=C^{2}$. Consider $f(z)=(\cos z, i \sin z)$. It is interesting to evaluate $\bar{f}$ along certain lines in $C^{2 \times 2}$ :

$$
\begin{aligned}
& \bar{f}\left(z \cdot\left[\begin{array}{ll}
1 & 0 \\
0 & 1
\end{array}\right]\right)=(\cos z, i \sin z), \quad \bar{f}\left(z \cdot\left[\begin{array}{cc}
0 & -i \\
-i & 0
\end{array}\right]\right)=(\exp z, 0), \\
& \bar{f}\left(z \cdot\left[\begin{array}{ll}
1 & 1 \\
1 & 1
\end{array}\right]\right)=\left(\frac{\exp 2 i z}{2}, \frac{\exp 2 i z}{2}\right), \quad \bar{f}\left(z \cdot\left[\begin{array}{ll}
0 & 0 \\
1 & 0
\end{array}\right]\right)=(1,0) .
\end{aligned}
$$

5. Let $E=A$, a commutative Banach algebra, and $F=C^{n}$. Then $G_{E F}=\left(A^{* *}\right)^{n \times n}$. Fix $\gamma_{1}, \ldots, \gamma_{n} \in A^{*}$, and suppose we are given the function $f(a)=\left(\gamma_{1}\left(a^{2}\right), \ldots, \gamma_{n}\left(a^{2}\right)\right)$. Then if $T \in\left(A^{* *}\right)^{n \times n}, \bar{f}(T)=T^{2}(\gamma)$, where the exponent indicates matrix multiplication (with the Arens product in $\left.A^{* *}\right)$, and $\gamma=\left(\gamma_{1}, \ldots, \gamma_{n}\right)$. 
One interesting feature of the extension map defined above is that it has a universal factorization property with respect to a certain class of extension maps: suppose $E$ is a closed subspace of a Banach space $G$, and that we have a continuous linear extension map

$$
e: H(E, F) \rightarrow H(G, F) \text {. }
$$

The formula $\beta(g)(u)=e(u)(g)$, for $u \in L(E, F)$ defines an element $\beta(g) \in$ $G_{E F}$ for every $g \in G$. We have, therefore, a map $\beta: G \rightarrow G_{E F}$. This map is analytic. We obtain the following proposition. The proof is simple.

Proposition 4. Define $\beta^{*}(h)=h \circ \beta$. Then the following are equivalent.

(a) The diagram

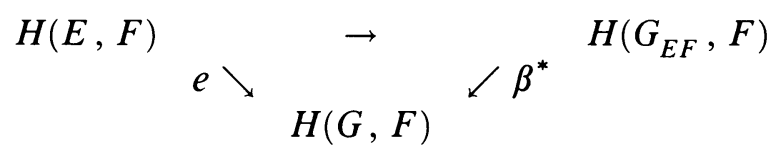

commutes.

(b) For all polynomials $P: E \rightarrow F, e(P)(g)=\bar{P}(\beta(g))$, for all $g \in G$.

(c) For all $\phi \in L_{s}^{k}(E, F)$ and all $g \in G, e(\hat{\phi})(g)=\overline{\beta(g)}^{k}(\phi)$.

If the equivalent conditions of the proposition hold, we say $e$ is an extension morphism. Some examples:

(i) Our extension $f \mapsto \bar{f}$ is a morphism.

(ii) If $h \in H(G, E)$, then $e(f)=f \circ h$ is a morphism. In this case, $\beta=\alpha \circ h$.

(iii) Let $F=C$, and let $E$ be such that the subalgebra of $H(E)$ generated by $E^{*}$ is dense $\left(c_{0}\right.$ and $l^{\infty}$, for example, see [6]). Then $e: H(E) \rightarrow H(G)$ is a morphism if and only if it is multiplicative.

Proof of (iii). $(\Rightarrow)$ Denote the map $f \mapsto \bar{f}$ by $\tau$. Being a morphism, $e$ factors through $\tau$, that is, $e=\beta^{*} \circ \tau$. But both $\beta^{*}$ and $\tau$ are multiplicative (we give a proof in $\S 3$ ).

$(\Leftrightarrow)$ Let $\gamma \in E^{*}$. For all $g \in G$, we have

$$
\beta^{*}(\bar{\gamma})(g)=\bar{\gamma}(\beta(g))=\beta(g)(\gamma)=e(\gamma)(g)
$$

so $\beta^{*} \circ \tau=e$ over $E^{*}$. Since both are linear and multiplicative, this equality is valid over the subalgebra of $H(E)$ generated by $E^{*}$. The conclusion follows from the continuity of all maps involved.

\section{EXTENSION OF THE DEFINITION TO ARBITRARY ANALYTIC FUNCTIONS}

We have extended a function $f \in H(E, F)$ to a function $\bar{f} \in H\left(G_{E F}, F\right)$ by extending its differentials at 0 and using the Taylor series expansion around this point. One might, however, want to define the extension from a different starting point, and indeed, if the domain of $f$ is not a ball, or even if the domain is $E$ but the radii of convergence are finite, one is forced to do so at different points of this domain. In order to extend our definition, we first prove a lemma which will be of use later. Recall that given a function $f$ analytic 
near $a, r_{a}$ denotes the radius of uniform convergence of $f$ at $a$. We drop the assumption that $f$ be bounded on bounded subsets of $E$.

Lemma 1. Let $a \in E$, and let $f$ be an $F$-valued function, analytic at $a$. Then, for any $p \geq 0$, and any $S \in G_{E F}$ such that $\|S-a\|<r_{a} / e$, we have

$$
\sum_{k \geq p} \frac{\overline{S-a}(k-p)}{(k-p) !}\left(D^{k} f(a)\right) \in L_{s}^{p}(E, F) \text {. }
$$

Proof. Indeed, for each $k, D^{k} f(a) \in L_{s}^{k}(E, F)$, so $\overline{S-a}^{(k-p)}\left(D^{k} f(a)\right) \in$ $L_{s}^{p}(E, F)$. We need only verify the absolute convergence of the series.

$$
\sum_{k \geq p}\left\|\frac{\overline{S-a}(k-p)}{(k-p) !}\left(D^{k} f(a)\right)\right\| \leq \sum_{k \geq p}\left\|\frac{1}{(k-p) !} D^{k} f(a)\right\|\|S-a\|^{k-p}
$$

but

$$
\begin{aligned}
& \underset{k}{\limsup }\left\|\frac{1}{(k-p) !} D^{k} f(a)\right\|^{1 / k-p} \\
& \quad=\underset{k}{\limsup }\left(\frac{k !}{(k-p) !}\right)^{1 / k-p}\left(\left\|\frac{1}{k !} D^{k} f(a)\right\|^{1 / k}\right)^{k / k-p} \\
& \quad \leq \limsup _{k}\left(\frac{k !}{(k-p) !}\right)^{1 / k-p}\left(\left(\frac{k^{k}}{k !} \|\left.\frac{1}{k !} D^{k} f(a)\right|_{\wp}\right)^{1 / k}\right)^{k / k-p} \\
& \quad=\frac{e}{r_{a}} .
\end{aligned}
$$

So the series converges if $\|S-a\|<r_{a} / e$.

Definition 3. Let $f$ be an $F$-valued function, analytic at $a \in E$. For $S \in G_{E F}$ such that $\|S-a\|<r_{a} / e$, define

$$
\begin{aligned}
\bar{f}(S) & =\sum_{k \geq 0} \frac{1}{k !} \overline{D^{k} f(a)}(S-a, \ldots, S-a) \\
& =\sum_{k \geq 0} \frac{\overline{S-a}}{k !}\left(D^{k} f(a)\right)
\end{aligned}
$$

Note that the series converges to an element of $F$ because of Lemma 1. Of course if $f$ is analytic at $x$ and $y$, and $S$ is sufficiently close to both points, we could have a problem of definition. We will show that there is no such problem.

Lemma 2. Let $a \in E$ and let $f$ be an $F$-valued function, analytic at $a$. Then for $S \in G_{E F}$ such that $\|S-a\|<r_{a} / e$, and $T \in G_{E F}$ such that $\bar{S} \circ \bar{T}=\bar{T} \circ \bar{S}$, we have

for all $p \geq 0$.

$$
D^{p} \bar{f}(S)(T, \ldots, T)=\bar{T}^{p}\left(\sum_{k \geq p} \frac{\overline{S-a}(k-p)}{(k-p) !}\left(D^{k} f(a)\right)\right)
$$


Proof. We proceed by induction in $p$. For $p=0$, this is simply the definition of $\bar{f}(S)$. Suppose then that $p>0$. Then

$D^{p} \bar{f}(S)(T, \ldots, T)=\lim _{\lambda \rightarrow 0} \frac{1}{\lambda}\left[D^{p-1} \bar{f}(S+\lambda T)(T, \ldots, T)-D^{p-1} \bar{f}(S)(T, \ldots, T)\right]$

Now $\overline{S+\lambda T}=\bar{S}+\lambda \bar{T}$ commutes with $\bar{T}$, so we may write

$$
\begin{aligned}
& =\bar{T}^{(p-1)} \lim _{\lambda \rightarrow 0} \frac{1}{\lambda}\left[\left(\sum_{k \geq p-1} \frac{\overline{S-a+\lambda T^{(k-p+1)}}}{(k-p+1) !}-\sum_{k \geq p-1} \frac{\overline{S-a}^{(k-p+1)}}{(k-p+1) !}\right)\left(D^{k} f(a)\right)\right] \\
& =\bar{T}^{(p-1)} \lim _{\lambda \rightarrow 0} \frac{1}{\lambda}\left[\left(\sum_{k \geq p} \frac{1}{(k-p+1) !} \sum_{i=1}^{k-p+1}\left(\begin{array}{c}
k-p+1 \\
i
\end{array}\right) \overline{S-a}^{(k-p+1-i)} \lambda^{i} \bar{T}^{i}\right)\left(D^{k} f(a)\right)\right] \\
& =\bar{T}^{(p-1)} \lim _{\lambda \rightarrow 0}\left[\left(\sum_{k \geq p} \frac{1}{(k-p+1) !} \sum_{i=1}^{k-p+1}\left(\begin{array}{c}
k-p+1 \\
i
\end{array}\right) \overline{S-a}^{(k-p+1-i)} \lambda^{(i-1)} \bar{T}^{i}\right)\left(D^{k} f(a)\right)\right] \\
& =\bar{T}^{(p-1)}\left(\sum_{k \geq p} \frac{k-p+1}{(k-p+1) !} \overline{S-a}^{(k-p)} \bar{T}\left(D^{k} f(a)\right)\right) \\
& =\bar{T}^{p}\left(\sum_{k \geq p} \frac{\overline{S-a}^{(k-p)}}{(k-p) !}\left(D^{k} f(a)\right)\right)
\end{aligned}
$$

and we are done.

Note that if $S=z \in E$, and $T=y \in E$, we have

$$
D^{p} f(z)(y, \ldots, y)=D^{p} \bar{f}(z)(y, \ldots, y)=\bar{y}^{p}\left(\sum_{k \geq p} \frac{\overline{z-a}^{(k-p)}}{(k-p) !}\left(D^{k} f(a)\right)\right)
$$

so

$$
D^{p} f(z)=\sum_{k \geq p} \frac{\overline{z-a}^{(k-p)}}{(k-p) !}\left(D^{k} f(a)\right) .
$$

We can now prove that the definition of $\bar{f}$ does not depend on the point $a$. This will depend on the partial commutativity property of Proposition 2: $\bar{a} \circ \bar{T}=$ $\bar{T} \circ \bar{a}$ for all $a \in E$ and $T \in G_{E F} . B(a, \rho)$ will denote the open ball of $G_{E F}$ of radius $\rho$ centered at $a$.

Theorem 1. $\bar{f}$ is well defined.

Proof. Suppose $x$ and $y$ are points of analyticity of $f$, such that the balls $B\left(x, r_{x} / e\right)$ and $B\left(y, r_{y} / e\right)$ have nonempty intersection. There are two definitions for the extension of $f$. Call them $F_{x}$ and $F_{y}$. We will show that they coincide on an open subset of $G_{E F}$. Let

$$
z=\frac{r_{y} x+r_{x} y}{r_{x}+r_{y}}
$$


and take $T \in B\left(x, r_{x} / e\right) \cap B\left(y, r_{y} / e\right)$. Then

$$
\begin{aligned}
\|x-z\| & =\frac{1}{r_{x}+r_{y}}\left\|\left(r_{x}+r_{y}\right) x-r_{y} x-r_{x} y\right\| \\
& =\frac{r_{x}}{r_{x}+r_{y}}\|x-y\| \leq \frac{r_{x}}{r_{x}+r_{y}}(\|x-T\|+\|T-y\|) \\
& <\frac{r_{x}}{r_{x}+r_{y}} \cdot \frac{r_{x}+r_{y}}{e}=\frac{r_{x}}{e} .
\end{aligned}
$$

And also,

$$
\|y-z\|<\frac{r_{y}}{e} .
$$

Since $z \in E, \bar{z} \circ \bar{T}=\bar{T} \circ \bar{z}$ for all $T \in G_{E F}$, so by Lemma 2, for all $p \geq 0$

$$
\begin{aligned}
D^{p} F_{x}(z)(T, \ldots, T) & =\bar{T}^{k}\left(\sum_{k \geq p} \frac{\overline{z-x}(k-p)}{(k-p) !}\left(D^{k} f(x)\right)\right) \\
& =\bar{T}^{k}\left(D^{p} f(z)\right)=\overline{D^{p} f(z)}(T, \ldots, T)
\end{aligned}
$$

and also,

$$
D^{p} F_{y}(z)(T, \ldots, T)=\overline{D^{p} f(z)}(T, \ldots, T) .
$$

Then for all $S$ in a sufficiently small neighborhood of $z$ in $G_{E F}$ we have

$$
\begin{aligned}
F_{x}(S) & =\sum_{k \geq 0} \frac{1}{k !} D^{k} F_{x}(z)(S-z, \ldots, S-z) \\
& =\sum_{k \geq 0} \frac{1}{k !} \overline{D^{k} f(z)}(S-z, \ldots, S-z) \\
& =\sum_{k \geq 0} \frac{1}{k !} D^{k} F_{y}(z)(S-z, \ldots, S-z) \\
& =F_{y}(S)
\end{aligned}
$$

so $F_{x}=F_{y}$.

Therefore, if $U \subset E$ is the domain of definition of an $F$-valued analytic function $f$, we may extend $f$ around all points of $U$, obtaining a function $\bar{f}$ defined on

$$
\widetilde{U}=\bigcup_{a \in U} B\left(a, \rho_{a}\right),
$$

where $\rho_{a}$ is the radius of uniform convergence of $\bar{f}$ at $a$. We know that, in general, this is at least $r_{a} / e$. Note that functions with the same domain of definition may have different radii of convergence, so $\widetilde{U}$ depends on $f$. For this reason we have no extension map as that of $\S 1$; the extensions of different functions have varying domains. Note also that the natural domain of definition for $\bar{f}$ may be larger than $\widetilde{U}$. We have the following examples. 
Examples. 6. Take $E=c_{0}$ and $F=C$. Then $G_{E F}=l^{\infty}$. It is well known [14] that the analytic function $f: c_{0} \rightarrow C$, given by

$$
f(x)=\sum_{k \geq 0} x_{1} \cdots x_{k}
$$

though analytic on all $c_{0}$, has radius of uniform convergence $r_{x}=1$ for all $x \in c_{0}$. Our extension gives $\bar{f}: \tilde{c}_{0} \rightarrow C$,

$$
\bar{f}(S)=\sum_{k \geq 0} S_{1} \cdots S_{k}
$$

where

$$
\tilde{c}_{0}=\left\{S \in l^{\infty}:\|S-x\|<1 \text {, for some } x \in c_{0}\right\} .
$$

Should we extend $g \in H\left(c_{0}\right)$, however, we would obtain $\bar{g} \in H\left(l^{\infty}\right)$.

7. Suppose $E=C$. Then $G_{E F}=B(F)$. Take $f=a \cdot h$, where $h: U \rightarrow C$. In this case

$$
\widetilde{U}=\{T \in B(F): \operatorname{sp}(T) \subset B \subset U, \text { for some ball } B\}
$$

whereas we know, because of the functional calculus, that $\bar{f}$ may actually be extended to the larger open set

$$
\{T \in B(F): \operatorname{sp}(T) \subset U\} .
$$

\section{A characterization of the ARon-Berner extension}

In what follows we restrict ourselves to the case $F=C$. We first give a characterization of this extension in terms of continuity properties of first order differentials.

Theorem 2. Let $g$ be any element of $H\left(E^{* *}\right)$ such that $\left.g\right|_{E}=f$. Then the following are equivalent:

(i) For all $x \in E, D g(x)$ is weakly continuous; and for all $z \in E^{* *}$ and $\left(x_{\alpha}\right) \subset E$ converging weakly to $z, D g(z)\left(x_{\alpha}\right) \rightarrow D g(z)(z)$.

(ii) $g=\bar{f}$.

Proof. Suppose (i) holds. Writing $g(z+\lambda L)$ and $g(z)$ around $x$, and doing as in Lemma 2 , one easily sees that, for any $x \in E$,

$$
D g(z)(L)=\sum_{k \geq 1} \frac{1}{(k-1) !} D^{k} g(x)(z-x, \ldots, z-x, L) .
$$

Now take $x \in E, L \in E^{* *}$, and $\left(x_{\alpha}\right) \subset E$ converging weakly to $L$, with $\left\|x_{\alpha}\right\| \leq\|L\|$ (Goldstine's theorem [9]). We want to prove the following: for all $n \in N$ there is a subnet $\left(x_{\alpha_{i}}\right)$ of $\left(x_{\alpha}\right)$ such that, for $k \leq n$,

$$
D^{k} g(x)\left(L, \ldots, L, x_{\alpha_{i}}\right) \rightarrow D^{k} g(x)(L, \ldots, L, L) .
$$

We proceed by induction on $n$ : 
For $n=1$, this is due to the weak continuity of $D g(x)$.

For $n>1$, we have $D^{k} g(x)\left(L, \ldots, L, x_{\alpha_{i}}\right) \rightarrow D^{k} g(x)(L, \ldots, L, L)$ for all $k \leq n-1$. Since $\left\{D^{n} g(x)\left(L, \ldots, L, x_{\alpha_{i}}\right)\right\}$ is a bounded subset of $C$, we may choose an accumulation point $\tau_{n}$ and a subnet of $\left(x_{\alpha_{i}}\right)$, which we will denote $\left(x_{r}\right)$, such that $D^{n} g(x)\left(L, \ldots, L, x_{r}\right) \rightarrow \tau_{n}$. Since $\left(x_{r}\right) \subset E$ and $x_{r} \rightarrow L$ weakly, $\left(x+\lambda x_{r}\right) \subset E$ and $x+\lambda x_{r} \rightarrow x+\lambda L$ weakly, for any $\lambda \in C$. Hence, by (i)

$$
D g(x+\lambda L)\left(x+\lambda x_{r}\right) \rightarrow D g(x+\lambda L)(x+\lambda L)
$$

by linearity,

$$
D g(x+\lambda L)\left(\lambda x_{r}\right) \rightarrow D g(x+\lambda L)(\lambda L)
$$

Then, using (1)

$$
\begin{aligned}
D g( & +\lambda L)\left(\lambda x_{r}\right)-D g(x+\lambda L)(\lambda L) \\
& =\sum_{k \geq 1} \frac{1}{(k-1) !}\left[D^{k} g(x)\left(\lambda L, \ldots, \lambda L, \lambda x_{r}\right)-D^{k} g(x)(\lambda L, \ldots, \lambda L, \lambda L)\right] \\
& =\sum_{k \geq 1} \frac{1}{(k-1) !}\left[D^{k} g(x)\left(L, \ldots, L, x_{r}\right)-D^{k} g(x)(L, \ldots, L, L)\right] \lambda^{k} .
\end{aligned}
$$

Taking the limit over $r$,

$$
0=\frac{\tau_{n}-D^{n} g(x)(L, \ldots, L)}{(n-1) !} \lambda^{n}+\lambda^{n+1} h_{n}(\lambda),
$$

where

$$
\begin{aligned}
h_{n}(\lambda)=\lim _{r}\left(\sum _ { k \geq n + 1 } \frac { 1 } { ( k - 1 ) ! } \left[D^{k} g(x)\left(L, \ldots, L, x_{r}\right)\right.\right. & \\
& \left.\left.-D^{k} g(x)(L, \ldots, L, L)\right] \lambda^{k-(n+1)}\right) .
\end{aligned}
$$

Since for all nonzero $\lambda \in C$

$$
D^{n} g(x)(L, \ldots, L)-\tau_{n}=\lambda(n-1) ! h_{n}(\lambda),
$$

we will have $D^{n} g(x)\left(L, \ldots, L, x_{r}\right) \rightarrow \tau_{n}=D^{n} g(x)(L, \ldots, L, L)$ if we show that $\left|h_{n}\right|$ is bounded in some neighborhood of 0 . Indeed, for any $r$ we have

$$
\begin{array}{rl}
\mid D^{k} & g(x)\left(L, \ldots, L, x_{r}\right)-D^{k} g(x)(L, \ldots, L, L) \mid \\
& =\left|D^{k} g(x)\left(L, \ldots, L, x_{r}-L\right)\right| \\
& \leq\left\|D^{k} g(x)\right\|\|L\|^{k-1}\left\|x_{r}-L\right\| \\
& \leq 2\left\|D^{k} g(x)\right\|\|L\|^{k} .
\end{array}
$$


So,

$$
\begin{aligned}
\left|h_{n}(\lambda)\right| & \leq \sum_{k \geq n+1} \frac{2\left\|D^{k} g(x)\right\|}{(k-1) !}\|L\|^{k}|\lambda|^{k-(n+1)} \\
& =2\|L\|^{n+1} \sum_{j \geq 0} \frac{\left\|D^{n+1+j} g(x)\right\|}{(n+j) !}\|L\|^{j}|\lambda|^{j}
\end{aligned}
$$

which, doing as in Lemma 1 , can be shown to be bounded near 0 . This proves (2). We now prove, for all $x \in E, L \in E^{* *}$, and $k \in N$,

$$
D^{k} g(x)(L, \ldots, L)=\overline{D^{k} f(x)}(L, \ldots, L) .
$$

Again, by induction on $k$ :

For $k=1$, note that $\overline{D f(x)}$ is weakly continuous by definition, while $D g(x)$ is weakly continuous by (i). They both coincide with $D f(x)$ on $E$, so they are equal on $E^{* *}$.

For $k>1$, let $\left(x_{\alpha}\right) \subset E$, converging weakly to $L$. Then

$$
\begin{aligned}
D^{k} g & (x)\left(L, \ldots, L, x_{\alpha}\right) \\
& =\lim _{\lambda \rightarrow 0} \frac{1}{\lambda}\left[D^{k-1} g\left(x+\lambda x_{\alpha}\right)(L, \ldots, L)-D^{k-1} g(x)(L, \ldots, L)\right] \\
& =\lim _{\lambda \rightarrow 0} \frac{1}{\lambda}\left[\overline{D^{k-1} f\left(x+\lambda x_{\alpha}\right)}(L, \ldots, L)-\overline{D^{k-1} f(x)}(L, \ldots, L)\right] \\
& =\lim _{\lambda \rightarrow 0}(\bar{L} \circ \ldots \circ \bar{L}) \frac{1}{\lambda}\left[D^{k-1} f\left(x+\lambda x_{\alpha}\right)-D^{k-1} f(x)\right]
\end{aligned}
$$

by the continuity of $(\bar{L} \circ \ldots \circ \bar{L})$ and the existence of

$$
\lim _{\lambda \rightarrow 0} \frac{1}{\lambda}\left[D^{k-1} f\left(x+\lambda x_{\alpha}\right)-D^{k-1} f(x)\right]=D^{k} f(x)_{x_{\alpha}} \in L_{s}^{k-1}(E),
$$

we obtain

$$
\begin{aligned}
D^{k} g(x)\left(L, \ldots, L, x_{\alpha}\right) & =(\bar{L} \circ \cdots \circ \bar{L})\left(D^{k} f(x)_{x_{\alpha}}\right) \\
& =\left[(\bar{L} \circ \cdots \circ \bar{L})\left(D^{k} f(x)\right)\right]\left(x_{\alpha}\right) .
\end{aligned}
$$

Now taking limit over a suitable subnet $\left(x_{r}\right)$ of $\left(x_{\alpha}\right)$ we have, by (2)

$$
\begin{aligned}
D^{k} g(x)(L, \ldots, L, L) & =L\left((\bar{L} \circ \ldots \circ \bar{L})\left(D^{k} f(x)\right)\right) \\
& =(\bar{L} \circ \bar{L} \circ \ldots \circ \bar{L})\left(D^{k} f(x)\right) \\
& =\overline{D^{k} f(x)}(L, \ldots, L, L) .
\end{aligned}
$$

Finally, $g=\bar{f}$ : just taken any $x \in E$, and $L \in E^{* *}$. Then

$$
g(x+L)=\sum_{k \geq 0} \frac{1}{k !} D^{k} g(x)(L, \ldots, L)=\sum_{k \geq 0} \frac{1}{k !} \overline{D^{k} f(x)}(L, \ldots, L)=\bar{f}(x+L) .
$$


For the other implication, suppose we have (ii). As in (1), one obtains

$$
\left.D \bar{f}(z)(T)=\sum_{k \geq 1} \frac{1}{k !} \overline{D^{k} f(0)}(z, \ldots, z, T)+\cdots+\overline{D^{k} f(0)}(T, z, \ldots, z)\right),
$$

only now the terms on the right are not, in general, equal, for $\overline{D^{k} f(0)}$ is not symmetric. But suppose $z \in E$, and $\left(T_{\alpha}\right) \subset E^{* *}$ converges weakly to $T$. Then $\overline{D^{k} f(0)}\left(z, \ldots, T_{\alpha}, \ldots, z\right)$ are all equal to $\overline{D^{k} f(0)}\left(T_{\alpha}, z, \ldots, z\right)$ which, being weakly continuous of its first variable, converges to $\overline{D^{k} f(0)}(T, z, \ldots, z)$. This, in turn, is independent of the order of $z, T$, because $z \in E$. So, we have that

$$
D \bar{f}(z)\left(T_{\alpha}\right)=\sum_{k \geq 1} \frac{1}{(k-1) !} \overline{D^{k} f(0)}\left(T_{\alpha}, z, \ldots, z\right)
$$

converges to

$$
\sum_{k \geq 1} \frac{1}{(k-1) !} \overline{D^{k} f(0)}(T, z, \ldots, z)=D \bar{f}(z)(T),
$$

so $D \bar{f}(z)$ is weakly continuous if $z \in E$. This is the first part of (i). Now suppose $z \in E^{* *}$, and $\left(x_{\alpha}\right) \subset E$ converges weakly to $z$. Then

$$
\overline{D^{k} f(0)}\left(z, \ldots, x_{\alpha}, \ldots, z\right)
$$

does not depend on the order of $z, x_{\alpha}$, because $x_{\alpha} \in E$. Then

$$
D \bar{f}(z)\left(x_{\alpha}\right)=\sum_{k \geq 1} \frac{1}{(k-1) !} \overline{D^{k} f(0)}\left(x_{\alpha}, z, \ldots, z\right)
$$

converges to

$$
\sum_{k \geq 1} \frac{1}{(k-1) !} \overline{D^{k} f(0)}(z, \ldots, z)=D \bar{f}(z)(z)
$$

which is the second part of (i).

Note that one may replace $E$ by a ball $B \subset E$ and $E^{* *}$ by a ball $B^{* *} \subset E^{* *}$ of equal radius. The following two examples should put condition (i) above in proper perspective.

Examples. 8. If $z$ is in $E^{* *}$, but not in $E, D \vec{f}(z)$ need not be weakly continuous, even at $z$ : Take $E=A \times A$, where $A$ is a commutative Banach algebra, such that the Arens product is not commutative. Recall that the Arens product is not weakly continuous of the second variable, so we can take $\left(S_{i}\right) \subset A^{* *}$ converging weakly to $S$, and $T \in A^{* *}$ such that $T S_{i}$ does not converge to $T S$. Fix $\gamma \in A^{*}$ such that $\left(T S_{i}\right)(\gamma)$ does not converge to $(T S)(\gamma)$, and fix $f: E \rightarrow C$ defined by $f(a, b)=\gamma(a b)$. Then $z_{i}=\left(S_{i}, T\right)$ converges weakly to $z=(S, T)$, and

$$
\begin{aligned}
& D \bar{f}(z)\left(z_{i}\right)-D \bar{f}(z)(z)=D \bar{f}(S, T)\left(S_{i}, T\right)-D \bar{f}(S, T)(S, T) \\
& \quad=\frac{1}{2}\left(S T+T S+T S_{i}+S_{i} T-S T-T S-T S-S T\right)(\gamma) \\
& \quad=\frac{1}{2}\left(\left(S_{i} T-S T\right)+\left(T S_{i}-T S\right)\right)(\gamma) .
\end{aligned}
$$


Now, $S_{i} T-S T$ converges weakly to 0 , so $D \bar{f}(z)\left(z_{i}\right)$ converges to $D \bar{f}(z)(z)$ if and only if $\left(T S_{i}\right)(\gamma)$ converges to $(T S)(\gamma)$, which is not the case. Hence $D \bar{f}(z)$ is not weakly continuous at $z$.

9. Weak continuity of $D g(x)$ for all $x \in E$ is not enough to guarantee $g=\bar{f}$, where $f=\left.g\right|_{E}$ : Let $E=A \times A$ as above, and $g(S, T)=(S T)(\gamma)$. We know from Example 1 that $\bar{f}(S, T)=\left(\frac{S T+T S}{2}\right)(\gamma)$. Fix $x=(a, b) \in E$. Then

$$
D g(x)(U, V)=(a V+U b)(\gamma)=(V a+U b)(\gamma),
$$

which is weakly continuous of $(U, V)$. However, $g$ is not $\bar{f}$.

We proceed now with some corollaries of the theorem.

Corollary 1. If $f_{1}, \ldots, f_{n} \in H(E)$, and $G \in H\left(C^{n}\right)^{k}$ is such that $G\left(f_{1}, \ldots, f_{n}\right)$ $=0$, then $G\left(\bar{f}_{1}, \ldots, \bar{f}_{n}\right)=0$.

Proof. Write $G=\left(G_{1}, \ldots, G_{k}\right)$, and consider, for $i=1, \ldots, k$, the functions $F_{i}: E^{* *} \rightarrow C$ given by

$$
F_{i}(T)=G_{i}\left(\bar{f}_{1}(T), \ldots, \bar{f}_{n}(T)\right) .
$$

Then $F_{i} \in H\left(E^{* *}\right)$, and for all $z \in E^{* *}$

$$
D F_{i}(z)=D G_{i}\left(\bar{f}_{1}(z), \ldots, \bar{f}_{n}(z)\right) \circ\left[D \bar{f}_{1}(z) \times \cdots \times D \bar{f}_{n}(z)\right]
$$

has the same continuity properties as the $D \bar{f}_{j}(z)$. But $F_{i}$ is zero over $E$, so Theorem 2 says that $F_{i}$ is the canonical extension of zero to $E^{* *}$. Hence $F_{i}=0$ for $i=1, \ldots, k$; so $G\left(\bar{f}_{1}, \ldots, \bar{f}_{n}\right)=0$.

Corollary 2. $H(E) \rightarrow H\left(E^{* *}\right)$ given by $f \mapsto \bar{f}$ is an algebra morphism.

Proof. We know it is continuous and linear, so we need only prove it is multiplicative. For this, just apply Corollary 1, where the function $G: C^{3} \rightarrow C$ is

$$
G(x, y, w)=w-x y
$$

and $f_{1}, \ldots, f_{n}$ are $f, g, f \cdot g$ for arbitrary $f$ and $g$ in $H(E)$.

We end with a short comment on the relationship between the Aron-Berner extension and an old problem of duality in spaces of holomorphic functions.

Suppose $\Omega$ is a domain of the complex plane. One has the following duality between functions analytic on $\Omega$, and germs of analytic functions on $\left(S^{2}-\Omega\right)$, where $S^{2}$ is the Riemann sphere.

$$
\langle f, g\rangle=\frac{1}{2 \pi i} \int_{\Gamma} f(\lambda) g(\lambda) d \lambda,
$$

where $\Gamma$ is a curve that encircles $\left(S^{2}-\Omega\right)$, contained in the domains of both $f$ and $g$. This was first observed by Sebastiao e Silva [15] and by Kothe [12]; and there have been various generalizations by Grothendieck [11], Tillman [16], and Gauthier and Rubel [10]. Note that when $\Omega=C$ one has a correspondence 
between $H(C)$ and $O_{\infty}$ (or $H(C)_{0}$ : germs of analytic functions at zero). On a general Banach space $E$, this correspondence generalizes to a map

$$
h: H(E)^{*} \rightarrow H\left(E^{*}\right)_{0}
$$

defined as follows: denote by $B_{r}$ the open ball of radius $r$ in $E$, and by $B_{s}^{*}$ the open ball of radius $s$ in $E^{*}$. For all $r, s$ such that $r \cdot s<1$, one has

$$
h_{r s}: H\left(B_{r}\right)^{*} \rightarrow H\left(B_{s}^{*}\right)
$$

defined by

$$
h_{r s}(L)(\gamma)=L\left(f_{\gamma}\right)
$$

where $f_{\gamma}(x)=(1-\gamma(x))^{-1}$. Letting $r$ grow to infinity and $s$ decrease to zero, one obtains the continuous monomorphism $h: H(E)^{*} \rightarrow H\left(E^{*}\right)_{0}$. In a completely analogous manner one defines

$$
k:\left(H\left(E^{*}\right)_{0}\right)^{*} \rightarrow H\left(E^{* *}\right) .
$$

The relationship between these maps and the Aron-Berner extension is given by the commutativity of the diagram

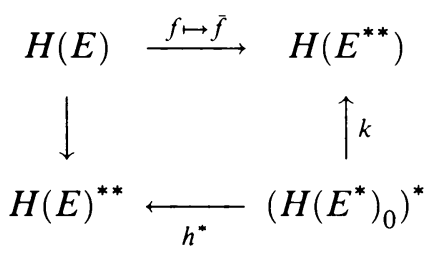

where the vertical arrow on the left is the canonical inclusion.

\section{REFERENCES}

1. R. Arens, Operations induced in function classes, Monatsh. Math. 55 (1951), 1-19.

2. __ The adjoint of a bilinear operation, Proc. Amer. Math. Soc. 2 (1951), 839-848.

3. R. Aron and P. Berner, A Hahn-Banach extension theorem for analytic mappings, Bull. Soc. Math. France 106 (1978), 3-24.

4. P. Boland, Holomorphic functions on nuclear spaces, Trans. Amer. Math. Soc. 209 (1975), 275-281.

5. S. B. Chae, Holomorphy and calculus in normed spaces, Marcel Dekker, 1985.

6. B. Cole and T. Gamelin, Representing measures and Hardy spaces for the infinite polydisk algebra, Proc. London Math. Soc. 53 (1986), 112-142.

7. A. Davie and T. Gamelin, A theorem on polynomial-star approximation, Proc. Amer. Math. Soc. (to appear).

8. S. Dineen, Holomorphically complete locally convex topological vector spaces, Séminaire Pierre Lelong: Analyse 1971/72, Lecture Notes in Math., vol. 332, Springer-Verlag, 1973, pp. 77-111.

9. N. Dunford and J. Schwartz, Linear operators. Part I, Wiley, 1964.

10. P. Gauthier and L. Rubel, Holomorphic functionals in open Riemann surfaces, Canad. J. Math. 28 (1976), 885-888.

11. A. Grothendieck, Sur certaines espaces de fonctiones holomorphes, J. Reine Angew. Math. 192 (1953), 35-64. 
12. G. Kothe, Dualität in der Funktionentheorie, J. Reine Angew. Math. 191 (1953), 30-49.

13. L. Moraes, A Hahn-Banach extension theorem for some holomorphic functions, Complex Analysis, Functional Analysis and Approximation Theory (ed. by J. Mujica), North-Holland Math. Stud. 125, North-Holland, 1986, pp. 205-220.

14. L. Nachbin, Topology on spaces of holomorphic mappings, Ergeb. Math. Grenzgeb., vol. 47, Springer-Verlag, 1969.

15. J. Sebastiao e Silva, As funções analiticas e a analise functional, Portugal. Math. 9 (1950), $1-130$.

16. G. Tillman, Dualität in der Funktionentheorie auf Riemannschen Flächen, J. Reine Angew. Math. 195 (1956), 76-101.

Department of Mathematics, University of California at los Angeles, Los Angeles, CALIFORNia 90024

Current address: Centre de Recerca Matemàtica, Apartat 50-08193 Bellaterra, (Barcelona) Spain 